\title{
INTRODUCTION
}

La transition énergétique des territoires impose de relever les défis cognitifs, organisationnels, informationnels et techniques qui contribueront demain à la construction d'un nouveau paradigme énergétique et à intégrer ainsi les mutations sociétales, technologiques et comportementales qui permettent d'en comprendre les mécanismes et les dynamiques.

Parmi les facteurs de réussite identifiés, sont avancées la nécessaire mise en cohérence des politiques publiques, l'articulation des échelles d'analyse et de décision et la compréhension des systèmes d'acteurs. La participation des habitants et plus largement des usagers, tout comme la transparence attendue de la part des opérateurs énergétiques, conditionnent notamment la richesse des données recueillies tout autant que les éventuelles solutions apportées aussi bien individuellement que collectivement. Dans ce contexte, les visions et approches souvent sectorielles et/ou techniciennes (approches dites « en silo ») cèdent peu à peu la place à une réelle prise de conscience de la complexité des problématiques énergétiques, entraînant la multiplication des pratiques et des projets interdisciplinaires.

Pourtant, alors même que les interactions font système, nous peinons encore à mettre en œuvre les solutions conceptuelles, méthodologiques, voire techniques, permettant de les capter et de les maîtriser. Les solutions passent alors par un décloisonnement disciplinaire et informationnel, indispensable tant les processus sont imbriqués. Cependant, dans le domaine énergétique, les systèmes d'information (SI) restent essentiellement centrés sur des données de consommation et/ou de production attachées à des dispositifs techniques. En dépit de leur qualité et de leur intérêt, ces informations/ données/mesures fournissent une connaissance souvent partielle du fonctionnement énergétique des territoires et, finalement, assez peu des comportements, cycles de vie, interactions et contextes territoriaux de production, de distribution, de consommation et d'usages. Cela est dû, en grande partie, aux difficultés à mobiliser, au-delà des corpus classiques, des données plus ou moins (in)formelles (crowdsourcing, données issues de réseaux mobiles, d'enquêtes de terrain, données open source, déclaratives issues de réseaux sociaux, etc.). Les modèles socio-cognitifs et les conflits que leur mise en œuvre peut générer, soulèvent des questionnements réflexifs en termes de métacognition, tant du point de vue des fournisseurs de données que de leurs utilisateurs. Par ailleurs, les représentations transmises à travers la diversité des sources, des données, mais surtout des modèles socio-culturels véhiculés, rendent difficiles les approches transversales et l'analyse des interactions entre les systèmes impliqués. 
En favorisant les échanges d'expériences et de points de vue autour d'une problématique généralement abordée de manière sectorielle, ce numéro se veut résolument pluridisciplinaire. Il ambitionne d'apporter des contributions pertinentes aux grandes questions et défis informationnels et cognitifs que nous posent la complexité et la nécessaire intégration des systèmes énergétiques/territoriaux en jeu et les informations qui leurs sont attachées. Comment favoriser l'intégration et l'exploitation conjointe de l'ensemble de ces informations spatiotemporelles à des fins opérationnelles : diagnostic, suivi, aide à la décision, prospective et, à terme, régulation ? Quelles articulations entre les dispositifs informationnels existants du local au «global »? Quel(s) cadre(s) sémantique(s) ? Quelles solutions conceptuelles (modèles, langages, etc.), méthodologiques et technologiques mettre en œuvre ? Quelle faisabilité ? Quels enseignements tirer des réalisations et comment accroître la performance des systèmes d'information et mettre en œuvre de véritables approches d'intelligence énergétique territoriale ?

Les contributions qui composent ce numéro apportent un éclairage pertinent à ces différentes questions.

Ainsi, l'article de Léone Alix Mazaud, Charlotte Suaud, Sarah Marquet, MarieHélène de Sède-Marceau, Matthieu Noucher, Hadrien Hainaut et Boris Bailly " Planification territoriale durable. Opportunités offertes par les données et les outils », propose, pour commencer, un état des lieux des outils et données mobilisés en France dans le cadre de la planification territoriale, et analyse, sur la base d'une proposition de typologie, les distorsions existantes entre les attentes en matière d'aide à la décision et les solutions apportées.

Nathalie Molines et Carine Henriot, dans leur article « Apports et limites de la thermographie aérienne comme outil de diagnostic de la performance énergétique d'un parc résidentiel. Exemple de l'agglomération de Compiègne », nous montrent l'intérêt, à partir d'une expérimentation de terrain, du couplage de différentes sources de données pour la production d'indicateurs composites conçus pour la sensibilisation et la planification énergétique.

Catherine Lavallez, Gilles Desthieux et Reto Camponovo s'attèlent, au-delà des données, sur la base d'un historique de la planification énergétique dans le canton de Genève, à la problématique des représentations spatialisées, à leur rôle et à celui des systèmes d'information géographique pour la planification énergétique territoriale, illustrant notamment les interactions entre évolution des politiques énergétiques territoriales et dispositifs informationnels et cognitifs.

Antoine Tabourdeau complète cette approche en abordant la problématique des acteurs de l'information énergétique et les enjeux autour de sa production. Dans son article intitulé « La construction d'observatoire du bois-énergie pour renouveler le rapport à la ressource. Les cas de l'Auvergne et de Rhônes-Alpes ", il nous montre combien, dans le domaine du bois-énergie, la recomposition du rôle des acteurs de l'information énergétique et, plus spécifiquement le rôle pris par les régions peut influer sur les représentations et les rapports à la ressource, dans un contexte de difficile articulation entre niveaux décisionnels. 
Enfin, Thomas Buhler et Philippe Signoret, en abordant la problématique du « captage » des comportements de mobilité dans le cadre de leur papier « Suivre les consommations énergétiques les déplacements quotidiens sur le temps long. Revisiter la méthode du panel à l'ère de la généralisation du smartphone », nous proposent, sur la base d'une expérimentation menée sur deux aires urbaines de Franche-Comté, d'intéressantes réflexions sur les perspectives théoriques et méthodologiques que nous offrent le captage de données énergétiques via les objets connectés et les défis posés tant par l'acceptabilité de ce type de dispositif que par l'intégration des données produites au sein de corpus plus larges.

Ce numéro spécial s'inscrit dans la suite de l'atelier organisé à Grenoble dans le cadre de SAGEO en novembre 2014 sur les enjeux informationnels et cognitifs de la transition énergétique. Nous tenions à remercier l'équipe qui a participé à l'organisation de cet évènement et tout particulièrement Yann Fléty et Philippe Signoret du Laboratoire ThéMA pour leurs précieuses contributions à la préparation de cette journée.

Marie-Hélène de SÈde-Marceau

ThéMA, UMR-6049, CNRS et Université de Bourgogne Franche-Comté

Sylvie Servigne

LIRIS UMR 5205, INSA de Lyon

\section{Comité de lecture}

Sabine Barles - Géographie Cités, Université Paris 1 Panthéon-Sorbonne Florence Brondeau - Université Paris-Sorbonne Sonia Chardonnel - PACTE, Université Joseph Fourier, Grenoble Gaëtan Cherix - Haute École d'Ingénierie de la HES-SO Valais-Wallis, Suisse Christophe Claramunt - Institut de Recherche de l'Ecole navale, Brest Paule-Annick Davoine - Lab. d'informatique de Grenoble, Grenoble-INP Institut polytechnique de Grenoble Thomas Devogele - Lab. d'informatique, BDTLN, Université de Tours Grégoire Feyt - PACTE, Université Joseph Fourier, Grenoble François Golay - Lab. de SIG, Institut d'ingénierie environnementale, École polytechnique de Lausanne, Suisse Didier Josselin - UMR Espace, Université D’Avignon et des Pays de Vaucluse Sylvie Lardon - UMR Métafort INRA, AgroParisTech, Clermont-Ferrand Thérèse Libourel - UMR ESPACE-DEV, Université de Montpellier 2

Taoufik Souami - LATTS, Université de Marne-la-Vallée Christiane Weber - UMR TETIS, AgroParisTech, Cirad, CNRS, Irstea Montpellier 
